\title{
NUMERICAL SOLUTION OF STOKES PROBLEM FOR FREE CONVECTION EFFECTS IN DISSIPATIVE DUSTY MEDIUM
}

\author{
V. VENKATARAMAN and K. KANNAN
}

Received 10 August 2004

\begin{abstract}
The flow past an infinite vertical isothermal plate started impulsively in its own plane in a viscous incompressible two-phase fluid has been considered by taking into account the viscous dissipative heat. The coupled nonlinear equations governing the flow are solved for fluid and particle phases by finite difference method. The velocity and temperature fields have been shown graphically for $G$ being positive for dusty air and it was observed that the same results hold for water. ( $G$ denotes the Grashof number and $G>0$ corresponds to cooling of the plate by free convection currents.) The results for $G<0$ (heating of the plate) have been verified and discussed. The numerical values of skin friction and the rate of heat transfer of dusty fluid are shown in tables. The effects of $\mathrm{G}$ and $\mathrm{E}$ (the Eckert number) on the flow field are discussed. It is observed that dusty fluid causes an increase in skin friction. The increase in mass concentration of dust particles decreases the heat transfer rate. The presence of inert particles does not admit the reverse type of flow even for large values of $t$.
\end{abstract}

2000 Mathematics Subject Classification: 76Txx.

1. Introduction. There have been numerous theoretical and experimental studies of heat and mass transfer induced by natural convection in fluids. The study of the flow of dusty fluids is of practical importance, particularly through packed beds, sedimentation, environmental pollution, chemical reactors, combustion systems, pneumatic transport, and centrifugal separation of particles.

The Stokes problem on free convection effects for an infinite vertical plate in a viscous incompressible fluid has a variety of physical applications, for example, filtration process, the drying of porous materials in textile industries and so forth.

The flow of an incompressible fluid past an impulsively started infinite horizontal plate in its own plane was first studied by Stokes [15]. Kazakevich and Krapivin [5] have experimentally studied the aerodynamic resistance of a dusty gas flowing through a system of pipes and have shown that the resistance is less than that of clear gas. Saffman [9] found an explanation for why the reduction of viscosity in dust particles in any gas has much larger inertia than that in an equivalent volume of air. The relative motion of dust particles and air will dissipate energy because of the drag between particles and air, and thus energy is extracted from the system. Saffman verified the above hypothesis on studying the stability of a laminar flow by investigating the effects of dust particles on the critical Reynolds number for transition to turbulent flow. 
Marble [6] has made a comparative study of experimental and theoretical results on gas and particle temperature in a rocket nozzle and pointed out the accuracy of predicted results of particle lag in rocket nozzles. Mukherjee [7] made a critical review on heat transfer to flowing gas-solid mixtures by performing an experiment on the transportation of gas particles in air, through a tube having uniform heat flux. Depew showed that these equations are valid for $30 \mu \mathrm{m}$ spheres and Reynolds number (less than 30000). The results are applicable only to very dilute concentrations and to very small particles obeying Stokes' drag law. However, not many studies on free convection problems of two-phase fluid are reported in available literature. Investigations of free convection effects in viscous incompressible and compressible fluids with variable viscosity have been made by many authors. Illingwoth [4] considered the flow of a compressible gas with variable viscosity near an impulsively started vertical plate and solved by the method of successive approximation. Illiott [1] generalized Illingwoth's problem by considering time-dependent velocity and temperature of the plate but neglected the viscous dissipative heat. Stewardson [14] studied Stokes' problem for a semi-infinite plate by an analytical method and Hall [2] studied it by a finitedifference method (FDM) approach. All researchers considered the plate horizontal. Soundalgekar [12] gave the closed form solution of the Stokes problem. He solved it by considering a vertical infinite plate, by taking into account the free convection effects but neglecting viscous dissipative heat. The plate was assumed to be isothermal and the effects of heating or cooling of the plate on the flow were considered. The same author [10] gave a numerical solution to this problem, when viscous dissipative heat is considered, the problem being governed by a coupled nonlinear system of equations. Helmy [3] gave a brief account of free convection effects in dusty conducting fluids. In subsonic flow of an incompressible fluid, the heat due to viscous dissipation is present in a number of physical phenomena. Also in the case of fluids with high Prandtl number, viscous dissipative heat is always present even in slow motions. But in presence of viscous dissipative heat the mechanisms of fluid-particle interaction are yet to be studied. In the present study, the flow past an infinite vertical isothermal plate starting impulsively in its own plane in a viscous incompressible two-phase fluid has been considered by taking into account the viscous dissipative heat. The governing equations for convective flow are coupled and nonlinear. Hence it is not possible to get an analytic solution. The problem has been solved by FDM approach.

2. Formulation of the problem. Here the $x^{\prime}$-axis is taken along the infinite plate in vertical direction and the $y^{\prime}$-axis is taken normal to the plate. The temperature of the plate is the same as that of the fluid when $t^{\prime}<0$. The plate starts from rest and moves in its own plane with a velocity $u_{0}$ at $t^{\prime}>0$ and the temperature is instantaneously raised or lowered to $T_{w}^{\prime}$ and is kept constant. All the fluid properties are assumed to be constant except that the influence of density variation with temperature has been considered only in the body force term. The buoyancy force on the dust particles is neglected. Following Saffman's model [9] of a dusty fluid, the governing equations for two-dimensional incompressible flow given by Marble [6] are 


$$
\begin{gathered}
\operatorname{div} \bar{u}=0 \\
\rho\left[\frac{\partial \bar{u}}{\partial t}+(\bar{u} \cdot \nabla) \bar{u}\right]=-\nabla p+\nabla[\mu \nabla \cdot \bar{u}]+\bar{F}_{p}, \\
\rho c_{p}\left[\frac{\partial T}{\partial t}+(\bar{u} \cdot \nabla) T\right]=\bar{u}_{t}+Q_{p}+\phi_{f}, \\
\operatorname{div} \bar{u}_{p}=0 \\
\rho\left[\frac{\partial \bar{u}_{p}}{\partial t}+\left(\bar{u}_{p} \cdot \nabla\right) \bar{u}_{p}\right]=-\nabla p_{p}-\bar{F}_{p}, \\
\rho c_{s}\left[\frac{\partial T_{p}}{\partial t}+\left(\bar{u}_{p} \cdot \nabla\right) T_{p}\right]=\bar{u}_{s}-Q_{p},
\end{gathered}
$$

where the volume fraction and viscosity of the pseudofluid of solid particles have been neglected. Here $\bar{u}, T, p$, and $\rho$ are the velocity, temperature, pressure, and density of fluid, respectively, and a subscript $p$ denotes corresponding entities of particle phase. $\mu$ and $c_{p}$, respectively, are the viscosity and specific heat of fluid, $c_{s}$ being the specific heat of particles. $u_{t}$ and $u_{p}$ represent heat fluxes for fluid phase and for particle phase, respectively. $\varphi_{f}$ is the viscous dissipation of fluid and $F_{p}$ is the total fluid-particle interaction force per unit volume. If the Reynolds number based on the relative velocity of particle is less than unity, then the force accelerating the particle to the fluid speed is given by Stokes' law, that is, $6 \pi r_{p} \mu\left(\bar{u}_{p}-\bar{u}\right)$, where $r_{p}$ is the radius of a particle. If $N$ is assumed to be the number density of the particles, the total interaction force per unit volume is $F_{p}=6 \pi N r_{p} \mu\left(\bar{u}_{p}-\bar{u}\right)=\rho_{p}\left(\bar{u}_{p}-\bar{u}\right) / \tau_{m}$, where $\tau_{m}=m / 6 \pi \mu r_{p}$ is called relaxation time during which the velocity of the particle phase relative to that of the fluid phase is reduced to $(1 / e)$ times its initial value and $m$ is the mass of each particle. Similarly, the total thermal interaction between the fluid and the particle phase per unit volume is given by $Q_{p}=\rho c_{S}\left(T_{p}-T\right) / \tau_{\tau}$, and $\tau_{\tau}=m c_{S} / 4 \pi K r_{p}$ is the thermal relaxation time of particle phase (temperature of the particle phase relative to fluid is $(1 / e)$ times its initial value). In most studies of dusty fluids, some simplifying assumptions are usually made for dilute suspensions [8]. In our present study of free convective effects, we make the following assumptions.

(1) The number density $N$ (the number of dust particles per unit volume of the mixture) of the particle is constant.

(2) Boussinesq's approximation is valid.

(3) The dust particles are assumed to be spherical in shape, all having the same radius and mass and all being undeformable.

(4) The solid particles are sparsely distributed and they are noninteracting, so that the pressure locally has the same velocity vector and temperature.

Due to this assumption, deficiency of randomness in local particle motion, the pressure associated with the particle cloud is negligible. So, the fluid pressure $p$ will be the same as total pressure of the mixture. Since the plate is infinite in $x^{\prime}$-direction, all the flow quantities are functions of $y$ and $t$ only. So $u^{\prime} \equiv u^{\prime}\left(y^{\prime}, t^{\prime}\right), u_{p}^{\prime} \equiv u_{p}^{\prime}\left(y^{\prime}, t^{\prime}\right)$, $T^{\prime} \equiv T^{\prime}\left(y^{\prime}, t^{\prime}\right), T_{p}^{\prime} \equiv T_{p}^{\prime}\left(y^{\prime}, t^{\prime}\right)$, and $\gamma^{\prime}=\gamma_{p}^{\prime}=0$. Thus the continuity equations of fluid 
and particle phases are identically satisfied. Therefore the governing equations of motion of dusty, unsteady, viscous incompressible fluid (2.1) can be written as

$$
\begin{gathered}
\rho \frac{\partial u^{\prime}}{\partial t^{\prime}}=\mu \frac{\partial^{2} u^{\prime}}{\partial y^{\prime 2}}-\frac{\partial p^{\prime}}{\partial x^{\prime}}-\rho_{g}+K N_{0}\left(u_{p}^{\prime}-u^{\prime}\right), \\
\frac{\partial u_{p}^{\prime}}{\partial t^{\prime}}=-\frac{\left(u_{p}^{\prime}-u^{\prime}\right)}{T_{p}}, \\
\rho c_{p} \frac{\partial T^{\prime}}{\partial t^{\prime}}=K \frac{\partial^{2} T^{\prime}}{\partial y^{\prime 2}}+N_{0} m \frac{c_{s}\left(T_{p}^{\prime}-T^{\prime}\right)}{T_{T}}+\mu \gamma\left(\frac{\partial u^{\prime}}{\partial y^{\prime}}\right)^{2}, \\
\frac{\partial T_{p}^{\prime}}{\partial t^{\prime}}=\frac{T_{p}^{\prime}-T^{\prime}}{T_{T}},
\end{gathered}
$$

where $u^{\prime}\left(y^{\prime}, t^{\prime}\right), u_{p}^{\prime}\left(y^{\prime}, t^{\prime}\right)$ are the velocities of the fluid and particle, respectively, and $p^{\prime}$ is the pressure. In the free stream from (2.2), we get

$$
0=-\frac{\partial p^{\prime}}{\partial x^{\prime}}-\rho_{\infty} g
$$

Eliminating $-\partial p^{\prime} / \partial x^{\prime}$ from (2.2) and (2.6), we get

$$
\rho \frac{\partial u^{\prime}}{\partial t^{\prime}}=\mu \frac{\partial^{2} u^{\prime}}{\partial y^{\prime 2}}+g\left(\rho_{\infty}-\rho\right)=K N_{0}\left(u_{p}^{\prime}-u^{\prime}\right) .
$$

Here $\rho=$ constant $=\rho_{\infty}$ in all terms except the buoyancy term $g\left(\rho_{\infty}-\rho\right)$. From the equation of state, we have

$$
g\left(\rho_{\infty}-\rho\right)=g \beta \rho_{\infty}^{\prime}\left(T^{\prime}-T_{\infty}^{\prime}\right)
$$

From (2.7) and (2.8) we obtain

$$
\frac{\partial u^{\prime}}{\partial t^{\prime}}=\gamma \frac{\partial^{2} u^{\prime}}{\partial y^{\prime 2}}+g \beta\left(T^{\prime}-T_{\infty}^{\prime}\right)+\frac{K N_{0}}{\rho}\left(u_{p}^{\prime}-u^{\prime}\right)
$$

where $\gamma$ is the kinematics viscosity of the fluid and $\beta$ is the coefficient of volume expansion.

The initial conditions of the problem are given by

$$
u^{\prime}=u_{p}^{\prime}=0, \quad T=T_{p}^{\prime}=T_{\infty}^{\prime} \quad \text { for } t^{\prime} \leq 0,
$$


and the boundary conditions are given by

$$
\begin{gathered}
u^{\prime}=u_{0}, \quad T^{\prime}=T_{w}^{\prime} \quad \text { at } y^{\prime}=0 \\
u^{\prime}=0, \quad T^{\prime}=T_{\infty}^{\prime} \quad \text { at } y^{\prime}=\infty
\end{gathered} \text { for } t^{\prime}>0 .
$$

Using the nondimensional variables

$$
\begin{gathered}
y=\frac{u_{0} y^{\prime}}{\gamma}, \quad u=\frac{u^{\prime}}{u_{0}}, \quad u_{p}=\frac{u_{p}^{\prime}}{u_{0}}, \quad \theta=\frac{T^{\prime}-T_{\infty}^{\prime}}{T_{w}^{\prime}-T_{\infty}^{\prime}}, \\
\gamma=\frac{c_{s}}{c_{p}}, \quad t=\frac{t^{\prime} u_{0}^{2}}{\gamma}, \quad \rho=\frac{\mu c_{p}}{K}, \quad \mathrm{G}=\frac{\gamma g \beta\left(T_{w}^{\prime}-T_{\infty}^{\prime}\right)}{u_{0}^{3}}, \\
\alpha=\frac{N_{0} m}{\rho}, \quad \lambda=\frac{\tau_{p} u_{0}^{2}}{\gamma}, \quad \theta_{p}=\frac{T_{p}^{\prime}-T_{\infty}^{\prime}}{T_{w}^{\prime}-T_{\infty}^{\prime}}, \\
\mathrm{P}=\frac{\mu c_{p}}{k} \quad \text { (Prandtl number), } \quad \mathrm{E}=\frac{u_{0}^{2}}{c_{p}\left(T_{w}^{\prime}-T_{\infty}^{\prime}\right)} \quad \text { (Eckert number), }
\end{gathered}
$$

where $\alpha$ is the concentration parameter, $G$ is the Grashof number, $\lambda$ is the nondimensional relaxation time, $\mathrm{P}$ is the Prandtl number, and E is the Eckert number, (2.9), (2.3), (2.4), and (2.5) become

$$
\begin{gathered}
\frac{\partial u}{\partial t}=\mathrm{G} \theta+\frac{\partial^{2} u}{\partial t^{2}}+\frac{\alpha}{\lambda}\left(u_{p}-u\right), \\
\frac{\partial u_{p}}{\partial t}=-\frac{1}{\lambda}\left(u_{p}-u\right), \\
\mathrm{P} \frac{\partial \theta}{\partial t}=\frac{\partial^{2} \theta}{\partial y^{2}}+\operatorname{PE}\left(\frac{\partial u}{\partial y}\right)^{2}+\frac{2 \alpha}{3 \lambda}\left(\theta_{p}-\theta\right), \\
\mathrm{P} \frac{\partial \theta_{p}}{\partial t}=-\frac{2}{3 \gamma \lambda}\left(\theta_{p}-\theta\right),
\end{gathered}
$$

and the initial and boundary conditions become

$$
\begin{gathered}
u(y, t)=u_{p}(y, t)=0 \\
\theta(y, t)=\theta_{p}(y, t)=0 \\
u(0, t)=\theta(0, t)=1 \quad \text { for } t \leq 0, \\
\theta_{p}(0, t)=u_{p}(0, t)=1 \quad \text { for } t>0, \\
u(\infty, t)=\theta(\infty, t)=0, \\
\theta_{p}(\infty, t)=u_{p}(\infty, t)=0 .
\end{gathered}
$$

We solve (2.13), (2.14), (2.15), and (2.16) by FDMs. Replacing the partial differential coefficients occurring in the equations by their finite difference quotients, we have 
the following finite difference equations:

$$
\begin{aligned}
& \left(\frac{u_{i, j+1}-u_{i, j}}{\Delta t}\right)=\mathrm{G} \theta_{i, j}+\frac{u_{i+1, j}-2 u_{i, j}+u_{i-1, j}}{(\Delta y)^{2}}+\frac{\alpha}{\lambda}\left(u_{p(i, j)}-u_{(i, j)}\right), \\
& \left(\frac{u_{p_{(i, j+1)}}-u_{p_{(i, j)}}}{\Delta t}\right)=-\frac{1}{\lambda}\left(u_{p(i, j)}-u_{(i, j)}\right), \\
& \mathrm{P}\left(\frac{\theta_{(i, j+1)}-\theta_{(i, j)}}{\Delta t}\right)=\left(\frac{\theta_{(i+1, j)}-2 \theta_{(i, j)}+\theta_{(i-1, j)}}{(\Delta y)^{2}}\right)+\operatorname{PE}\left(\frac{u_{(i, j)}-u_{(i-1, j)}}{\Delta y}\right)^{2} \\
& +\frac{2 \alpha}{3 \lambda}\left(\theta_{p(i, j)}-\theta_{(i, j)}\right), \\
& \mathrm{P}\left(\frac{\theta_{p(i, j+1)}-\theta_{p(i, j)}}{\Delta t}\right)=-\left(\frac{2}{3 v \lambda}\right)\left(\theta_{p(i, j)}-\theta_{(i, j)}\right) .
\end{aligned}
$$

Here the index $i$ refers to $y$ and $j$ refers to $t$. $\Delta y$ is taken to be 0.1 .

From (2.17),

$$
\begin{aligned}
u(0,0) & =1, & & \theta(0,0)=1, \\
u_{p}(0,0) & =1, & & \theta_{p}(0,0)=1 .
\end{aligned}
$$

For all $i$ except $i=0$,

$$
\begin{aligned}
u(i, 0) & =1, & & \theta(i, 0)=1, \\
u_{p}(i, 0) & =1, & & \theta_{p}(i, 0)=1 .
\end{aligned}
$$

From the boundary conditions,

$$
\begin{aligned}
u(0, j) & =1, \quad \theta(0, j)=1, \\
u_{p}(0, j) & =1, \quad \theta_{p}(0, j)=1 \quad \forall j .
\end{aligned}
$$

According to analytical solutions of (2.13) for $\alpha=0$, we will regard $y=4.1$ as the point corresponding to $y=\infty$ and all of $u, u_{p}, \theta, \theta_{p}$ tend to zero at $y \sim 4$ for all values of $\mathrm{P}$ and G. Therefore we take

$$
u(41, j)=0, \quad \theta(41, j)=0, \quad u_{p}(41, j)=0, \quad \theta_{p}(41, j)=0 .
$$

This is true for all $j$ equivalent to boundary condition (2.19). The velocities at the end of the time step, namely, $u_{i, j+1}$ and $u_{p(i, j+1)}, i=1$ to 40 , are computed from (2.20) and (2.21) in terms of velocities and temperatures at points of the earlier time step. Similarly $\theta_{i, j+1}, \theta_{p(i, j+1)}$ are computed from (2.22) and (2.23). The procedure is repeated till $t=1$ $(j=400)$. The computations are carried out and the curves of $u$ and $u_{p}$ against $y$ are traced using MATLAB software. The numerical solution obtained for $\alpha=0$ is compared with the numerical solutions obtained by Soundalgekar [11] and there is perfect agreement. Besides, to ensure the accuracy of results, the solution obtained was compared with the analytic solution at $t=0.2,0.4$, and 0.5 . For the entire range of $y$ values an excellent agreement was found with maximum error less than one percent. The convergence was also tested for smaller values of $\Delta t$; namely, $\Delta t=0.002,0.001,0.003$. The changes in the results were found to be negligible. It has been shown by Soundalgekar [13] that $G<0$ corresponds to heating of the plate and $G>0$ corresponds to cooling of the plate. 


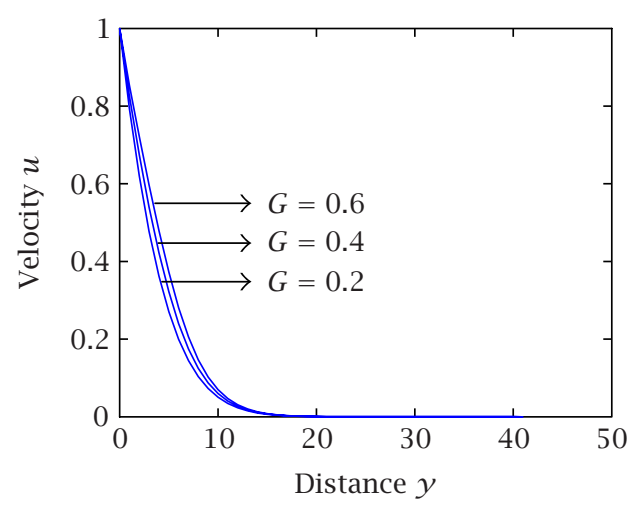

(a)

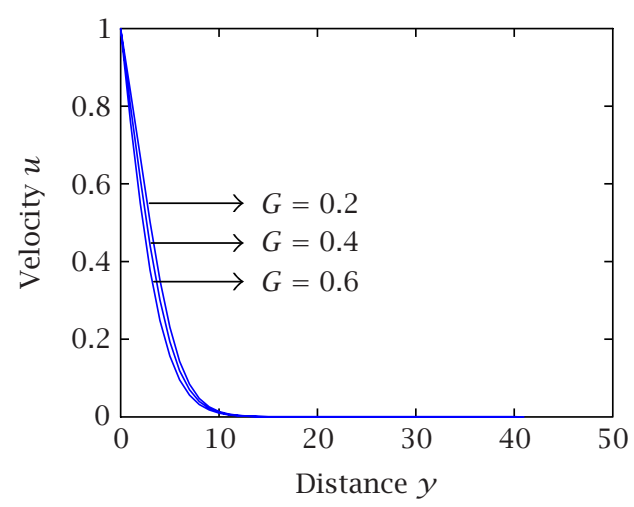

(c)

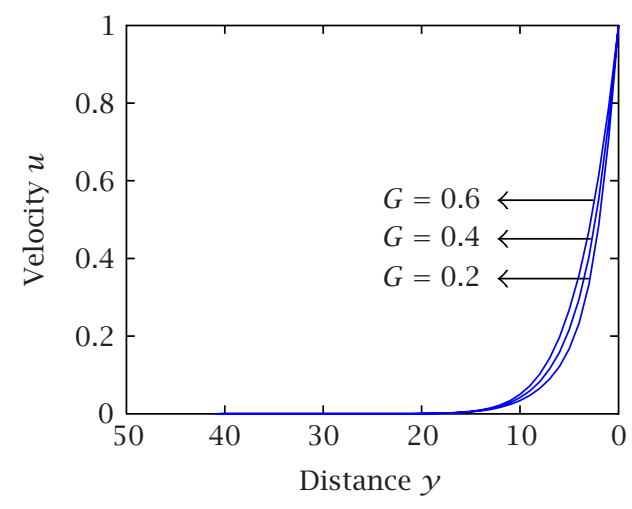

(b)

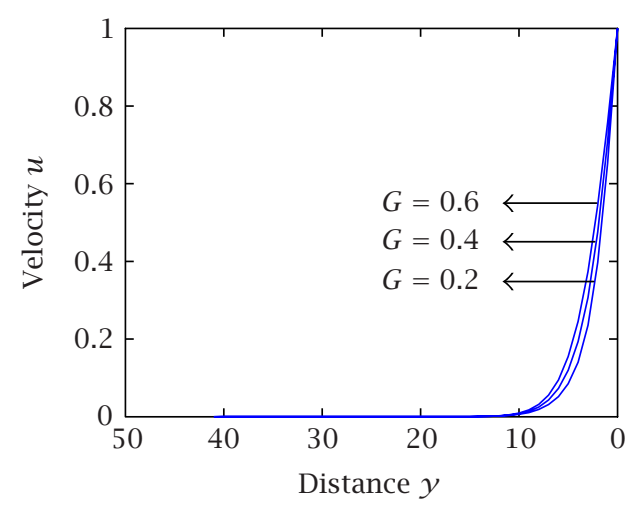

(d)

FiguRE 3.1. (a, b) Velocity profiles of fluid phase. (c, d) Velocity profiles of particle phase. (a, c) $G>0, E=0.2$; (b, d) $G<0, E=0.2$.

3. Results and discussion. The velocity profiles for both fluid and particle phase are depicted in Figure 3.1, for dusty air $(\mathrm{P}=0.71, \alpha=0.001)$, in the case of the plate being cooled by free convection currents. We observe that due to presence of viscous dissipative heat, there is a rise not only in velocity of air (fluid phase) but also in particle phase. But when compared to air, particle velocity is less. Also it follows from Figure 3.1 that greater cooling of the plate causes a rise in velocity. It has also been verified that the increase in time leads to a rise in velocity of both phases. We observe from Figure 3.2 that greater viscous dissipative heat causes more rise in velocity $(\mathrm{E}=0.2,0.5$, and 0.8 ) in both phases. Velocity profiles of both phases are drawn in Figure 3.3. For a slightly higher mass concentration parameter, $\alpha=0.002$, we find that an increase in Grashof number (greater than 0 ) causes an increase in velocity of both phases. It is interesting to infer from the same figure that closer are the velocity profiles of both the fluid and particle phases, though $\mathrm{G}$ takes values 0.2 and $0.9(\alpha=0.002, t=0.2)$. Also 


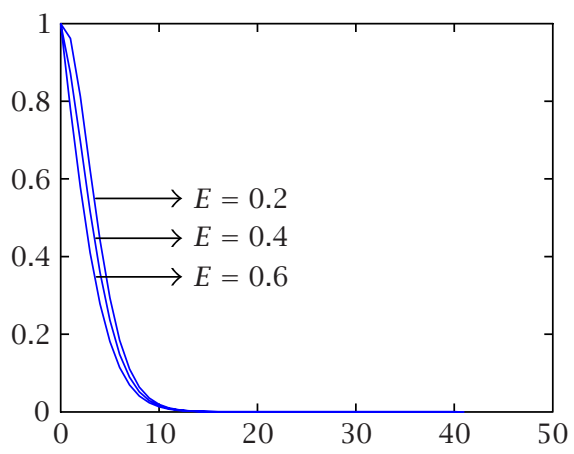

(a)

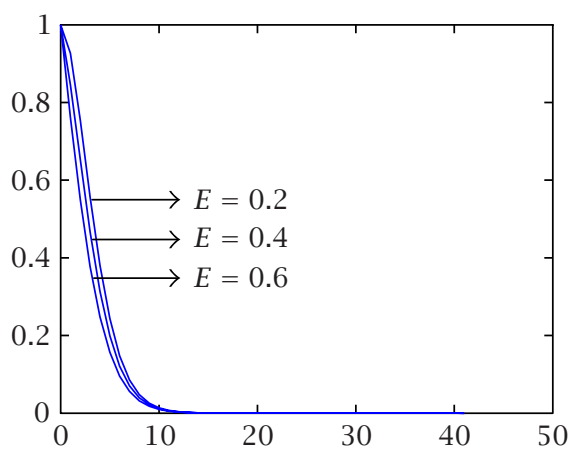

(c)

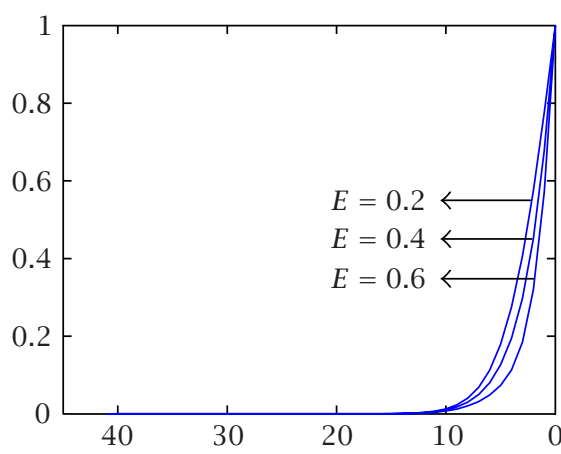

(b)

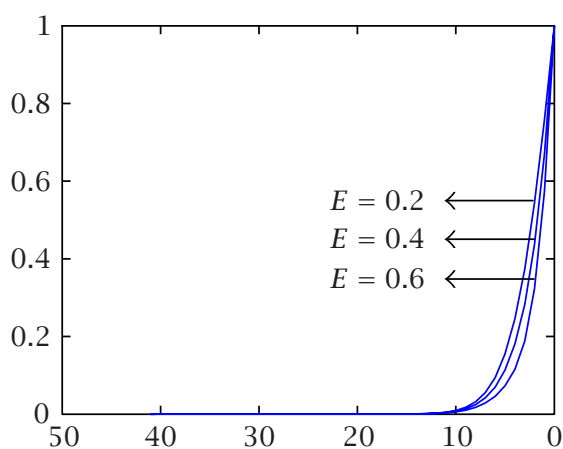

(d)

FiguRE 3.2. (a, b) Velocity profiles of fluid phase. (c, d) Velocity profiles for particle phase. (a, c) $G>0$; (b, d) $G<0$.

heating/cooling of the plate causes a small change in relaxation time of inert particle. It can be seen from Figure 3.4 that heating and cooling of the plate have almost the same effect on the velocities of both phases at $t=0.5$. From Figure 3.5 , for $\mathrm{G}<0$, greater viscous dissipative heat causes a fall in temperature not only in fluid phase but also in particle phase. Having studied velocity of fluid, we now calculate skin friction given by

$$
\tau=-\left(\frac{d u}{d y}\right)_{y=0} \text { where } \tau=\frac{\tau^{1}}{\rho u_{0}^{2}}
$$

By using Newton's interpolation formula of numerical differentiation and by taking five points, the values of $\tau$ for $\mathrm{P}=7$ and 0.71 (water and air) are calculated and entered in Tables 3.1 and 3.2, respectively. We observe from Tables 3.1 and 3.2 that skin friction decreases as time increases (for both air and water). Greater dissipative heat causes fall in skin friction. However, we find that a small increase of mass concentration causes increase in skin friction (Tables 3.1 and 3.4). For clean fluids, for $\mathrm{G}=1$ and $t=1$, skin friction is observed to be negative and hence possibility for reverse type of flow had 


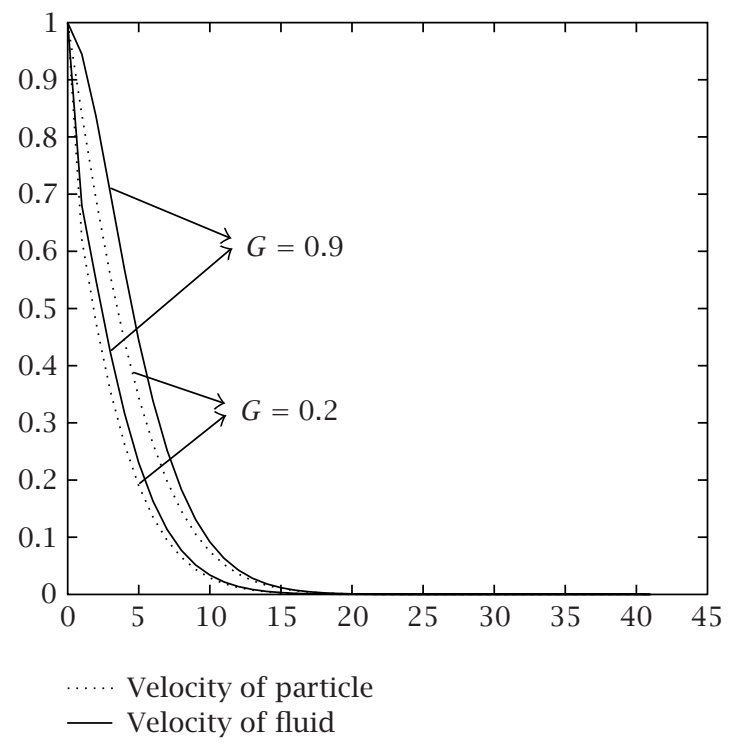

FIGURE 3.3. Velocity profiles of both particles for $G>0$.

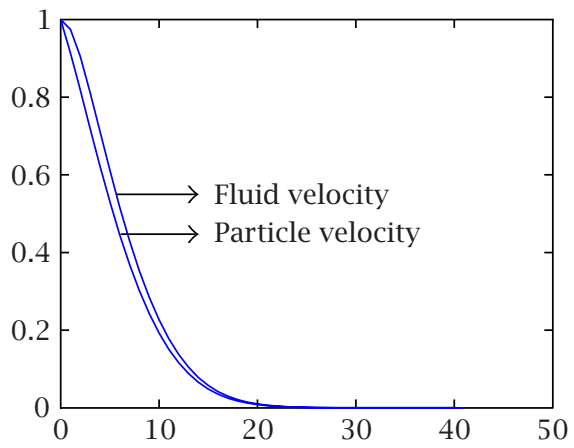

(a)

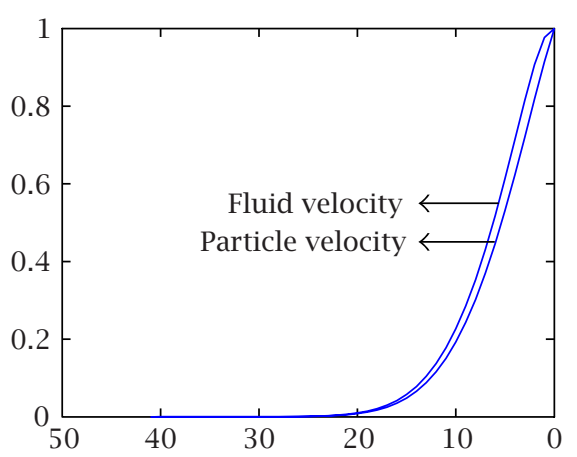

(b)

FIGURE 3.4. Velocity profiles of both phases at $t=0.5$. (a) $G>0, E=0.2$. (b) $G<0, E=0.2$.

been observed by Soundalgekar [13] for large values of $t$ when the plate is cooled. But in dusty fluids ( $\alpha=0.001 ; \alpha=0.0025$ ), we observe that skin friction is positive. So it is important to note that the presence of inert particles does not admit the reverse type of flow for large values of $t$. This is true in the cases of water and air. Also for dusty fluids it is true that skin friction is more in the case of water than in the case of air. Skin friction increases with temperature of the plate. The rate of heat transfer is given 


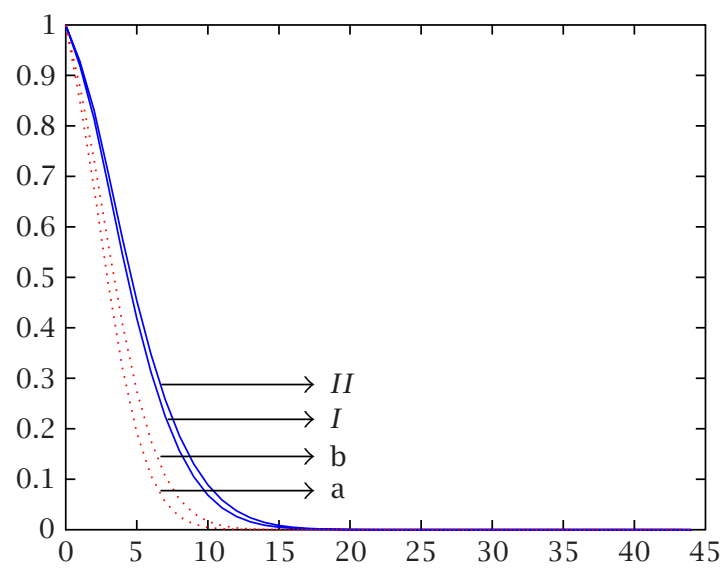

Fluid phase

$\begin{array}{cccccccc}t & G & E & & t & G & E & \\ 0.4 & -0.02 & 0.01 & I & 0.4 & -0.02 & 0.01 & \mathrm{a} \\ 0.4 & -0.02 & 0.04 & I I & 0.4 & -0.02 & 0.04 & \mathrm{~b}\end{array}$

FIGURE 3.5. Temperature profiles of fluid and particle phases.

TABLE 3.1. Values of $\tau$ and $q$; $\mathrm{P}=7.0$, water $(\alpha=0.0025, \lambda=0.025)$.

\begin{tabular}{c|c|ccc|ccc}
\hline \multirow{2}{*}{$\mathrm{G}$} & \multirow{2}{*}{$\mathrm{E} / \boldsymbol{t}$} & \multicolumn{3}{|c|}{$\boldsymbol{T}$} & & \multicolumn{2}{c}{$q$} \\
\cline { 3 - 7 } & & 0.2 & 0.5 & 1.0 & 0.2 & 0.5 & 1.0 \\
\hline 0.2 & 0 & 1.2369 & 0.7558 & 0.4930 & 3.3645 & 2.1152 & 1.4950 \\
0.2 & 0.01 & 1.2366 & 0.7554 & 0.4925 & 3.3274 & 2.0937 & 1.4805 \\
0.2 & 0.02 & 1.2364 & 0.7551 & 0.4921 & 3.2904 & 2.0722 & 1.4660 \\
-0.2 & 0 & 1.2917 & 0.8428 & 0.6163 & 3.3645 & 2.1152 & 1.4950 \\
-0.2 & -0.01 & 1.2915 & 0.8425 & 0.6158 & 3.4025 & 2.1379 & 1.5111 \\
-0.2 & -0.02 & 1.2913 & 0.8422 & 0.6154 & 3.4405 & 2.1606 & 1.5272 \\
0.7 & 0 & 1.1683 & 0.6469 & 0.3388 & 3.3645 & 2.1152 & 1.4950 \\
0.7 & 0.01 & 1.1675 & 0.6458 & 0.3373 & 3.3286 & 2.0951 & 1.4821 \\
0.7 & 0.02 & 1.1668 & 0.6447 & 0.3357 & 3.2926 & 2.0751 & 1.4693 \\
-0.7 & 0 & 1.3602 & 0.9517 & 0.7704 & 3.3645 & 2.1152 & 1.4950 \\
-0.7 & -0.01 & 1.3594 & 0.9508 & 0.7689 & 3.4037 & 2.1395 & 1.5134 \\
-0.7 & -0.02 & 1.3586 & 0.9494 & 0.7673 & 3.4428 & 2.1638 & 1.5318 \\
1.0 & 0 & 1.1272 & 0.5816 & 0.2463 & 3.3645 & 2.1152 & 1.4950 \\
1.0 & 0.01 & 1.1261 & 0.5800 & 0.2441 & 3.3292 & 2.0959 & 1.4829 \\
1.0 & 0.02 & 1.1250 & 0.5784 & 0.2419 & 3.2939 & 2.0766 & 1.4709 \\
-1.0 & 0 & 1.4014 & 1.0170 & 0.8629 & 3.3645 & 2.1152 & 1.4950 \\
-1.0 & -0.01 & 1.4002 & 1.0153 & 0.8606 & 3.4044 & 2.1406 & 1.5150 \\
-1.0 & -0.02 & 1.3991 & 1.0137 & 0.8583 & 3.4441 & 2.1659 & 1.5349 \\
\hline
\end{tabular}


TABLE 3.2. Values of $\tau$ and $q$; $\mathrm{P}=0.71$, air $(\alpha=0.001, \lambda=0.025)$.

\begin{tabular}{c|c|cccc|cccc}
\hline \multirow{2}{*}{$\mathrm{G}$} & \multirow{2}{*}{$\mathrm{E} / t$} & \multicolumn{5}{|c|}{$\tau$} & \multicolumn{4}{c}{$q$} \\
\cline { 3 - 9 } & & 0.2 & 0.5 & 0.7 & 1.0 & 0.2 & 0.5 & 0.7 & 1.0 \\
\hline 0.2 & 0 & 1.2098 & 0.7130 & 0.5733 & 0.5758 & 1.0643 & 0.6729 & 0.5686 & 0.5711 \\
0.2 & 0.01 & 1.2097 & 0.7129 & 0.5732 & 0.5757 & 1.0592 & 0.6699 & 0.5662 & 0.5687 \\
0.2 & 0.02 & 1.2096 & 0.7128 & 0.5731 & 0.5756 & 1.0541 & 0.6670 & 0.5638 & 0.5663 \\
-0.2 & 0 & 1.3188 & 0.8857 & 0.7777 & 0.5802 & 1.0643 & 0.6729 & 0.5686 & 0.5711 \\
-0.2 & -0.01 & 1.3188 & 0.8855 & 0.7775 & 0.7800 & 1.0697 & 0.6763 & 0.5716 & 0.5741 \\
-0.2 & -0.02 & 1.3187 & 0.8854 & 0.7774 & 0.7799 & 1.0751 & 0.6798 & 0.5745 & 0.5770 \\
0.7 & 0 & 1.0734 & 0.4971 & 0.3178 & 0.3203 & 1.0643 & 0.6729 & 0.5686 & 0.5711 \\
0.7 & 0.01 & 1.0732 & 0.4968 & 0.3174 & 0.3199 & 1.0596 & 0.6704 & 0.5667 & 0.5695 \\
0.7 & 0.02 & 1.0729 & 0.4964 & 0.3170 & 0.3195 & 1.0548 & 0.6679 & 0.5647 & 0.5672 \\
-0.7 & 0 & 1.4552 & 1.1015 & 1.0331 & 1.0356 & 1.0643 & 0.6729 & 0.5686 & 0.5711 \\
-0.7 & -0.01 & 1.4549 & 1.1011 & 1.0327 & 1.0352 & 1.0701 & 0.6770 & 0.5724 & 0.5749 \\
-0.7 & -0.02 & 1.4547 & 1.1007 & 1.0322 & 1.0347 & 1.0760 & 0.6811 & 0.5762 & 0.5787 \\
1.0 & 0 & 0.9916 & 0.3676 & 0.1645 & 0.1670 & 1.0643 & 0.6729 & 0.5686 & 0.5711 \\
1.0 & 0.01 & 0.9913 & 0.3671 & 0.1640 & 0.1665 & 1.0598 & 0.6706 & 0.5669 & 0.5694 \\
1.0 & 0.02 & 0.9909 & 0.3666 & 0.1634 & 0.1659 & 1.0552 & 0.6683 & 0.5651 & 0.5676 \\
-1.0 & 0 & 1.5370 & 1.2310 & 1.1864 & 1.1889 & 1.0643 & 0.6729 & 0.5686 & 0.5711 \\
-1.0 & -0.01 & 1.5366 & 1.2305 & 1.1857 & 1.1882 & 1.0704 & 0.6775 & 0.5730 & 0.5755 \\
-1.0 & -0.02 & 1.5362 & 1.2299 & 1.8510 & 1.1876 & 1.0765 & 0.6820 & 0.5773 & 0.5798 \\
\hline
\end{tabular}

TABLE 3.3. Values of $\tau$ and $q ; \mathrm{P}=0.71$, air $(\alpha=0.0025, \lambda=0.025)$.

\begin{tabular}{c|c|ccc|ccc}
\hline \multirow{2}{*}{$\mathrm{G}$} & \multirow{2}{*}{$\mathrm{E} / t$} & \multicolumn{3}{|c|}{$\tau$} & \multicolumn{3}{c}{$q$} \\
\cline { 3 - 7 } & & 0.2 & 0.5 & 0.7 & 0.2 & 0.5 & 0.7 \\
\hline 0.2 & 0 & 1.2459 & 0.7615 & 0.6288 & 3.3645 & 2.1152 & 1.7872 \\
0.2 & 0.01 & 1.2457 & 0.7612 & 0.6284 & 3.2730 & 2.0936 & 1.7694 \\
0.2 & 0.02 & 1.2455 & 0.7609 & 0.6280 & 3.2900 & 2.0720 & 1.7516 \\
-0.2 & 0 & 1.3007 & 0.8485 & 0.7318 & 3.3645 & 2.1152 & 1.7872 \\
-0.2 & -0.01 & 1.3005 & 0.8482 & 0.7315 & 3.4027 & 2.1380 & 1.8064 \\
-0.2 & -0.02 & 1.3003 & 0.8479 & 0.7311 & 3.4408 & 2.1608 & 1.8256 \\
0.7 & 0 & 1.1774 & 0.6527 & 0.5000 & 3.3645 & 2.1152 & 1.7832 \\
0.7 & 0.01 & 1.1766 & 0.6516 & 0.4987 & 3.2840 & 2.0950 & 1.7710 \\
0.7 & 0.02 & 1.1759 & 0.6505 & 0.4974 & 3.2923 & 2.0749 & 1.7547 \\
-0.7 & 0 & 1.3692 & 0.9573 & 0.8606 & 3.3645 & 2.1152 & 1.7872 \\
-0.7 & -0.01 & 1.3684 & 0.9562 & 0.8593 & 3.4038 & 2.1396 & 1.8083 \\
-0.7 & -0.02 & 1.3676 & 0.9550 & 0.8580 & 3.4431 & 2.1641 & 1.8294 \\
1.0 & 0 & 1.1363 & 0.5875 & 0.4227 & 3.3645 & 2.1152 & 1.7872 \\
1.0 & 0.01 & 1.1352 & 0.5859 & 0.4208 & 3.3290 & 2.0958 & 1.7718 \\
1.0 & 0.02 & 1.1341 & 0.5843 & 0.4190 & 3.2936 & 2.0765 & 1.7564 \\
-1.0 & 0 & 1.4103 & 1.0226 & 0.9379 & $3645 *$ & 2.1152 & 1.7872 \\
-1.0 & -0.01 & 1.4092 & 1.0209 & 0.9360 & $* 4046$ & 2.1407 & 1.8096 \\
-1.0 & -0.02 & 1.4080 & 1.0192 & 0.9341 & $* 4445$ & 2.1662 & 1.8319 \\
\hline
\end{tabular}


TABLE 3.4. Values of $\tau$ and $q$; $\mathrm{P}=7.0$, water $(\alpha=0.001, \lambda=0.025)$.

\begin{tabular}{|c|c|c|c|c|c|c|c|}
\hline \multirow{2}{*}{ G } & \multirow{2}{*}{$\mathrm{E} / t$} & \multicolumn{3}{|c|}{$T$} & \multicolumn{3}{|c|}{$q$} \\
\hline & & 0.2 & 0.5 & 0.7 & 0.2 & 0.5 & 0.7 \\
\hline 0.2 & 0 & 1.2189 & 0.7188 & 0.5783 & 1.0643 & 0.6729 & 0.5686 \\
\hline 0.2 & 0.01 & 1.2188 & 0.7186 & 0.5781 & 1.0592 & 0.6699 & 0.5662 \\
\hline 0.2 & 0.02 & 1.2187 & 0.7185 & 0.5780 & 1.0541 & 0.6669 & 0.5638 \\
\hline-0.2 & 0 & 1.3278 & 0.8913 & 0.7824 & 1.0643 & 0.6729 & 0.5686 \\
\hline-0.2 & -0.01 & 1.3277 & 0.8912 & 0.7822 & 1.0693 & 0.6764 & 0.5716 \\
\hline-0.2 & -0.02 & 1.3276 & 0.8910 & 0.7821 & 1.0752 & 0.6798 & 0.5746 \\
\hline 0.7 & 0 & 1.0827 & 0.5032 & 0.3231 & 1.0643 & 0.6729 & 0.5686 \\
\hline 0.7 & 0.01 & 1.0824 & 0.5029 & 0.3227 & 1.0595 & 0.6704 & 0.5667 \\
\hline 0.7 & 0.02 & 1.0822 & 0.5025 & 0.3223 & 1.0548 & 0.6780 & 0.5647 \\
\hline-0.7 & 0 & 1.4640 & 1.1068 & 1.0375 & 1.0643 & 0.6729 & 0.5686 \\
\hline-0.7 & -0.01 & 1.4637 & 1.1065 & 1.0371 & 1.0702 & 0.6770 & 0.5724 \\
\hline-0.7 & -0.02 & 1.4634 & 1.1061 & 1.0366 & 1.0760 & 0.6811 & 0.5762 \\
\hline 1.0 & 0 & 1.0010 & 0.3739 & 0.1700 & 1.0643 & 0.6729 & 0.5686 \\
\hline 1.0 & 0.01 & 1.0006 & 0.3734 & 0.1695 & 1.0597 & 0.6706 & 0.5669 \\
\hline 1.0 & 0.02 & 1.0003 & 0.3729 & 0.1689 & 1.0552 & 0.6683 & 0.5651 \\
\hline-1.0 & 0 & 1.5457 & 1.2362 & 1.1906 & 1.0643 & 0.6729 & 0.5686 \\
\hline-1.0 & -0.01 & 1.5453 & 1.2356 & 1.1899 & 1.0704 & 0.6775 & 0.5730 \\
\hline-1.0 & -0.02 & 1.5449 & 1.2351 & 1.1893 & 1.0766 & 0.6821 & 0.5774 \\
\hline
\end{tabular}

by

$$
q^{\prime}=-k\left(\frac{\partial T^{\prime}}{\partial y^{\prime}}\right)_{y=0}
$$

and in nondimensional form it is given by

$$
q=-\left(\frac{\partial \theta}{\partial y}\right)_{y=0}
$$

where

$$
q=\frac{\gamma q^{\prime}}{K u_{o}\left(T_{w}^{\prime}-T_{\infty}^{\prime}\right)}
$$

The numerical values of $q$ obtained for water and air are displayed in Tables 3.1, 3.2, 3.3, and 3.4 for $\alpha=0.0025$ and 0.001 . We include the points observed from Tables 3.1 to 3.4 regarding dusty fluids in concluding remarks.

4. Conclusions. (1) Heat transfer rate increases with greater cooling or heating of the plate. 
(2) But greater viscous dissipative heat causes a fall in the rate of heat transfer when $\mathrm{G}>0$ and a rise in the rate of heat transfer when $\mathrm{G}<0$.

(3) It is important to note that even a small increase in mass concentration of dust particles causes a fall in the rate of heat transfer.

(4) Mass concentration of dust particles plays a dominant role in changing the heat transfer rate of dusty fluids.

(5) For $G><0$, greater viscous dissipative heat causes more rise in velocity not only in fluid phase but also in particle phase.

(6) Greater heating of the plate causes a fall in velocity and greater cooling of the plate causes a rise in velocity in particle phase.

(7) Greater cooling increases the relaxation time of particle phase.

(8) At large $t$, heating and cooling of the plate have almost got the same effect for both phases.

(9) For $G<0$, greater viscous dissipative heat causes a fall in temperature in both phases.

(10) Presence of inert suspended Stokesian solid particles in the fluid does not admit reverse type of flow even for large values of $t$.

ACKNOWLEDGMENT. The authors wish to thank Dr. V. Ramamurthy, Professor and Head of SRM Deemed University for his critical comments towards the improvement of this paper.

\section{REFERENCES}

[1] L. Elliott, Unsteady laminar flow of gas near an infinite flat plate, Z. Angew. Math. Mech. 49 (1969), 647-652.

[2] M. G. Hall, The boundary layer over an impulsively started flat plate, Proc. Roy. Soc. London Ser. A 310 (1969), 401-414.

[3] K. A. Helmy, On free convection of a dusty conducting fluid, Indian J. Pure Appl. Math. 32 (2001), no. 3, 447-467.

[4] C. R. Illingworth, Unsteady laminar flow of gas near an infinite flat plate, Proc. Cambridge Philos. Soc. 46 (1950), 603-613.

[5] F. P. Kazakevich and A. M. Krapivin, Investigations of heat transfer and aerodynamical resistance in tube assemblies when the flow of gas is dust-laden, Izv. Vissh. Uchebn. Zavedenii Energetica 1 (1958), 101-107 (Russian).

[6] F. E. Marble, Dynamics of a gas containing small solid particles, Proceedings of the Fifth AGARD Combustion and Propulsion Colloqium (1962), Pergamon Press, New York, 1963, pp. 175-215.

[7] S. K. Mukherjee, Study of incompressible seperated flow problem in fluid mechanics, Ph.D. thesis, Indian Institute of Technology, Kharagpur, 1990.

[8] V. Ramamurthy, Study of some dusty flow problems without and with magnetic field, Ph.D. thesis, Indian Institute of Technology, Kharagpur, 1987.

[9] P. G. Saffman, On the stability of laminar flow of a dusty gas, J. Fluid Mech. 13 (1962), 120-128.

[10] V. M. Soundalgekar, Free convection effects on the oscillatory flow past an infinite, vertical, porous plate with constant suction-I, Proc. Roy. Soc. London Ser. A 333 (1973), 2536.

[11] _ Free convection effects on the oscillatory flow past an infinite, vertical, porous plate with constant suction-II, Proc. Roy. Soc. London Ser. A 333 (1973), 37-50. 
[12] __ Free convection effects on Stokes problem for an infinite vertical plane, J. Heat Trans. (Tr. Asme) 99c (1977), 499-501.

[13] V. M. Soundalgekar, J. P. Bhat, and M. Mohiuddin, Finite difference analysis of free convection effects on Stokes problem for a vertical plate in dissipative fluid, Internat. J. Engrg. Sci. 17 (1979), no. 12, 1283-1288.

[14] K. Stewartson, On the impulsive motion of a flat plate in a viscous fluid, Quart. J. Mech. Appl. Math. 4 (1951), 182-198.

[15] G. G. Stokes, On the effect of the internal friction of fluids on the motion of pendulums, Trans. Cambridge Philos. Soc. 9 (1851), 8-106.

V. Venkataraman: Shanmugha Arts, Science, Technology and Research Academy (SASTRA), Deemed University, Tirumalaisamudram, Thanjavur 613402, Tamil Nadu, India

E-mail address: mathvvr1@rediffmai 1 .com

K. Kannan: Shanmugha Arts, Science, Technology and Research Academy (SASTRA), Deemed University, Tirumalaisamudram, Thanjavur 613402, Tamil Nadu, India

E-mail address: dr_kkannan@yahoo.com 


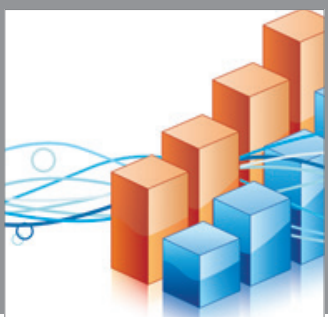

Advances in

Operations Research

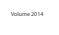

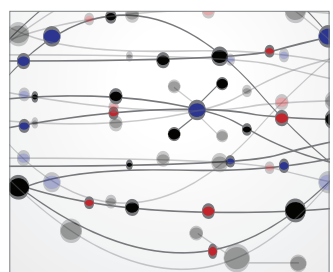

\section{The Scientific} World Journal
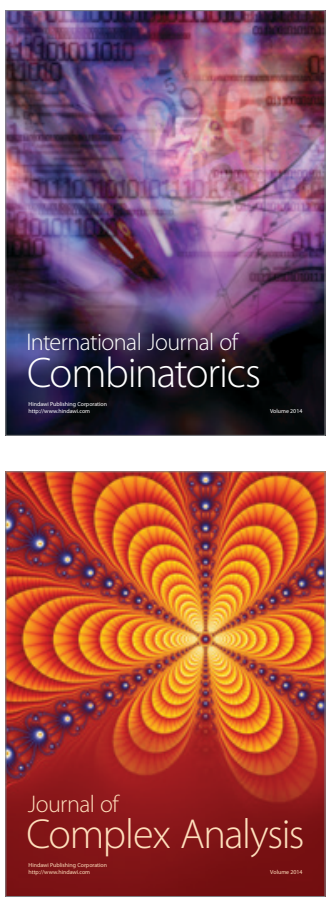

International Journal of

Mathematics and

Mathematical

Sciences
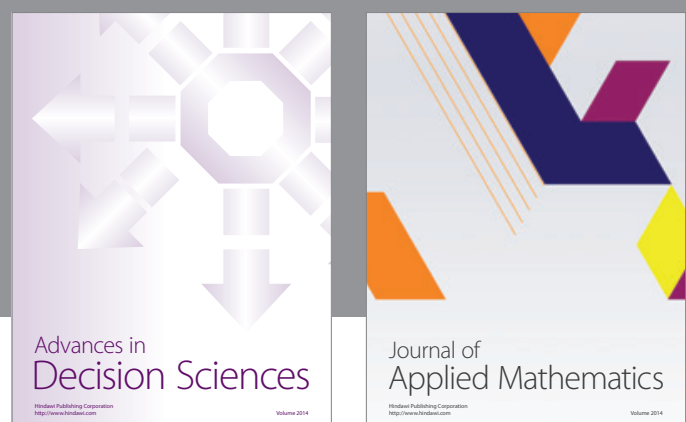

Journal of

Applied Mathematics
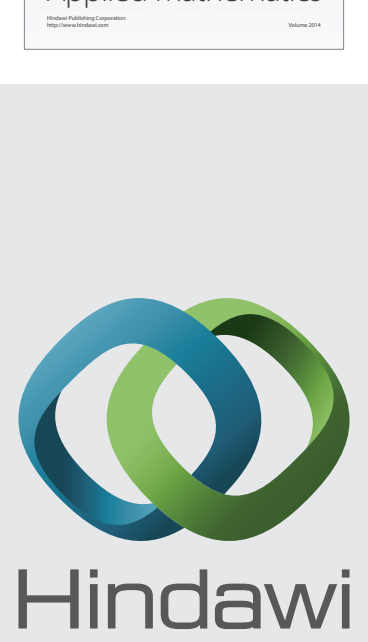

Submit your manuscripts at http://www.hindawi.com
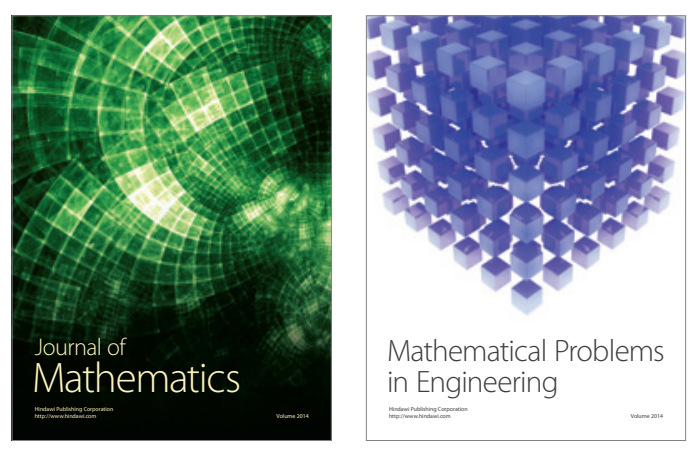

Mathematical Problems in Engineering
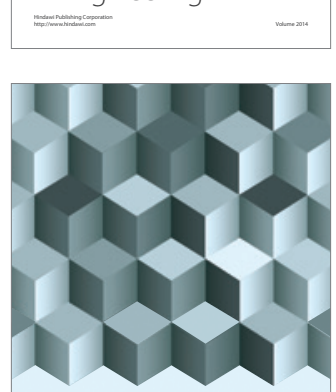

Journal of

Function Spaces
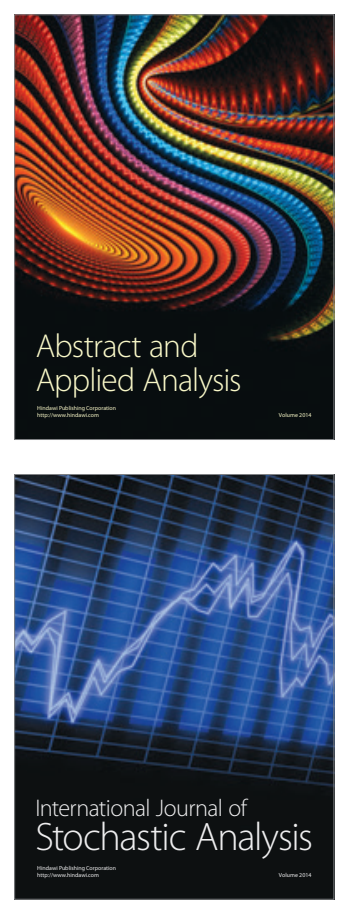

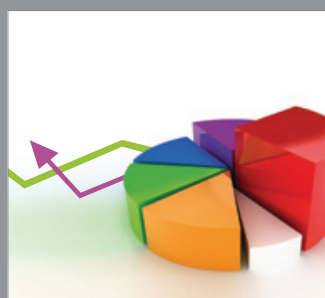

ournal of

Probability and Statistics

Promensencen
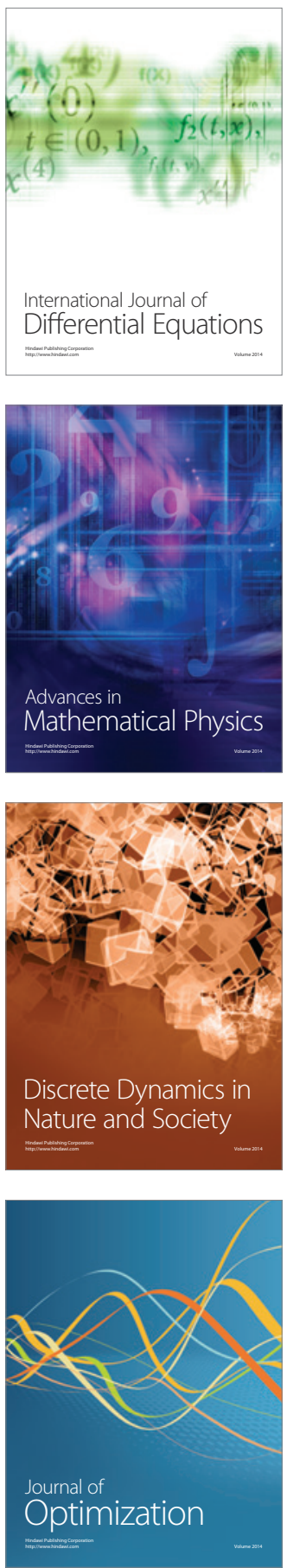